\title{
Ciclo de Mejora del Aprendizaje en Enseñanzas Técnicas de Introducción al Acondicionamiento Ambiental y a las Bases de los Sistemas Constructivos
}

\section{Learning Improvement Cycle on Technical Subjects related with Environmental Conditioning and Building Systems Introduction}

CARLOS RIVERA-GÓMEZ

ORCID: https://orcid.org/0000-0001-5324-068X

Universidad de Sevilla

E.T.S. de Arquitectura.

Departamento de Construcciones

Arquitectónicas I

crivera@us.es

Fecha de recepción: 20/09/19

Fecha de aceptación del artículo: 25/11/19

DOI: $h$ ttp://dx.doi.org/10.12795/9788447221912.112

Pp.: 2493-2511 
La necesidad de la adaptación de los recursos didácticos al perfil y las necesidades de los estudiantes de materias técnicas implica una revisión de los métodos y técnicas usados para favorecer el aprendizaje. La presente comunicación presenta un Ciclo de Mejora del Aprendizaje (CIMA) en el Aula en contenidos de acondicionamiento ambiental e introducción a los sistemas constructivos en la asignatura de Construcción I, del primer curso del Grado en Fundamentos de la Arquitectura en la Universidad de Sevilla, implantando un nuevo modelo docente que combina la resolución de problemas con la clase invertida. A través de un diseño metodológico adaptado a los contenidos se pretende alcanzar un aprendizaje más efectivo a través de la resolución de ejercicios prácticos en el aula, la discusión en grupo y su aplicación a casos reales de estudio. La metodología propuesta incorpora recursos didácticos desarrollados en anteriores CIMAs, incidiendo especialmente tanto en los criterios como en los instrumentos de evaluación.

Palabras clave: Construcción I, Arquitectura, Docencia universitaria, Experimentación docente universitaria, Aprendizaje basado en problemas.

\section{Abstract}

The requirement to adapt the didactic resources to the students' needs and profiles trained on technical subjects implies a review of the methods and techniques used to favour learning. The present communication shows a Learning Improvement Cycle (LIC) regarding environmental conditioning and introduction to construction systems contents in the subject of Construction I, belonging to the first year of the Architecture Degree in the University of Seville, implementing a new teaching model that combines the problem-based learning with the flipped classroom. Through a methodological design adapted to the contents, it is intended to achieve more effective learning through the resolution of practical exercises in the class, group discussion and its application to real case studies. The proposed methodology incorporates didactic resources developed in previous LICS, with special emphasis on both the criteria and the evaluation instruments.

Keywords: Construction I, Architecture, University teaching, University teaching experimentation, Problem-based learning. 


\section{Contexto de la Asignatura}

La asignatura en la que se ha aplicado el CIMA es Construcción 1 del Grado en Fundamentos de Arquitectura. Es una asignatura troncal y está situada en el primer cuatrimestre del primer año de carrera. Los contenidos de la asignatura están destinados a introducir al alumno en los rudimentos de la tecnología constructiva y su representación gráfica, así como en los aspectos relativos al acondicionamiento y las instalaciones de los edificios. El perfil de los alumnos es desigual, proviniendo de bachillerato en su mayoría, aunque también de ciclos formativos en edificación. Estos últimos abordan la asignatura con un dominio considerable de los recursos de representación gráfica constructiva. Los temas tratados durante el CIMA son los mismos que ya se abordaron el curso pasado 2018/19 con una ampliación relativa al contenido del segundo de dichos temas. En concreto se trata del tema: La Calidad del Aire interior y su control. Los objetivos de este tema son la intelección de los requisitos básicos de la ventilación de edificios y la lógica constructiva, de cálculo y de diseño aplicada a estos sistemas dentro del marco de la normativa. Y, por otra, el tema: Estructuras Horizontales Forjados Unidireccionales de Hormigón Armado, de este tema en el pasado CIMA se abarcó el apartado referente al replanteo inicial de dichos forjados, a esos contenidos y procedimientos se han añadido el presente curso 2019/20, dos sesiones centradas en la técnica de Aprendizaje Basado en Problemas (ABP) en los que se ha abordado la importancia de la definición constructiva de los forjados mediante el desarrollo de detalles constructivos de puntos singulares del forjado. Los objetivos generales de dicho tema son el conocimiento de los requisitos, funcionalidad, proceso constructivo y descripción gráfica de los elementos estructurales que integran un forjado unidireccional de hormigón armado. Se ha seleccionado dicho tema por la implicación constructiva que los sistemas de ventilación suponen en las soluciones de forjado. Considerando, para 
ambos temas, la necesidad del conocimiento de la normativa a través de los documentos básicos correspondientes del Código Técnico de la Edificación [1].

\section{Principios Didácticos}

En relación a los Principios Didácticos fundamentales que han primado en la concepción y desarrollo del CIMA han estado inspirados por las directrices enunciadas por Blain [2] y Finkel [3] y han sido de diversa indole. En primer lugar un Propósito General de Mejora respecto al CIMA impartido el curso anterior, evitando errores tácticos, de administración de tiempos y contenidos. Por otra parte también se ha tenido en consideración los relativos a la Exposición y Sistematización de Contenidos mediante el Mapa de Contenidos y Problemas del tema tratado. Asimismo se ha tenido en cuenta el principio de Vinculación de la Teoría con la Práctica. Bien sea presentando a los estudiantes ciertos hechos de la realidad conectados con el asunto estudiado (la investigación personal sobre sistemas de ventilación en sus casas o en la escuela de arquitectura) o bien proponiendo como ejemplo la solución de Problemas prácticos de la Vida Real. Se ha tenido presente el principio de Asequibilidad, tratando de acercar progresivamente al alumno a los aspectos más abstractos justificándolos en contenidos más directamente inteligibles y relacionados con su contexto habitual. También se han Jerarquizado los Contenidos, insistiendo en aquellos aspectos más complejos con objeto de que se fijen y consoliden, relegando a un segundo plano los menos importantes. La metodología ha hecho posible el principio de Equilibrar el Rol del Profesor y el Trabajo Independiente de los Estudiantes, permitiendo la motivación y activación de estos últimos y prestando la necesaria atención al cuidado de las condiciones externas del proceso. La figura del profesor ha tratado de actuar en este entorno garantizando el principio básico de Atención Individualizada del 
estudiante en la interacción con el grupo y con la clase en su conjunto.

\section{Diseño previo del CIMA}

El CIMA del curso 2019/20 se ha llevado a cabo en cinco sesiones de dos horas, hasta las diez horas presenciales a las que han sumado unas quince horas de trabajo en casa realizado de forma autónoma por el estudiante. Considerado de forma global el ciclo ha abordado veinticinco horas de trabajo a realizar durante tres semanas. Las fechas concretas que abarcará serán los días 21, 23, 28 y 30 de octubre y el 4 de noviembre de 2019. El Objetivo General del ciclo ha consistido en el aprendizaje de los conceptos teóricos, herramientas de cálculo y herramientas gráficas que permitan al alumno no sólo entender el sentido arquitectónico de los temas estudiados, sino solucionar mediante un lenguaje gráfico casos reales relativos a dichos temas.

Por su parte, los Objetivos Específicos han consistido en ayudar al estudiante en el desarrollo de sus aptitudes creativas. Favorecer la evaluación continua y la autoevaluación del propio estudiante. Favorecer el paso hacia trabajo autónomo al iniciar al alumno en el aprendizaje independiente. Analizar y resolver cuestiones propias de la práctica profesional. Acercar a los estudiantes al tipo de problemas que se encontrarán en el futuro. Facilitar el aprendizaje de competencias complejas asociadas a la resolución de problemas, el trabajo en equipo o la toma de decisiones. Fomentar el trabajo en grupo. Motivar al estudiante para que aprenda a debatir. Desarrollar las habilidades de comunicación del alumno. Y experimentar un aprendizaje y evaluación ligados a hechos reales.

Respecto a las cuestiones asociadas a la Metodología del CIMA se han considerado las siguientes: Analizar la 
jerarquización de contenidos y técnicas con el objeto de extraer de entre ellos los esenciales y favorecer su aprendizaje mediante las herramientas didácticas adecuadas. Realizar un diseño de las sesiones de trabajo en clase que incluya el material de aprendizaje necesario y una planificación del tiempo acorde con las respectivas actividades desarrolladas. Proporcionar al alumno las herramientas adecuadas para la resolución de las actividades planteadas para realizarse autónomamente fuera del aula. Posibilitar los medios que permitan una inmediata y adecuada valoración del aprendizaje del estudiante a lo largo del CIMA. Registrar el transcurso de las sesiones para poder obtener la información pertinente que permita analizar el CIMA en su conjunto como parte de una experiencia de mejora docente.

A continuación se muestra el diseño completo del Ciclo de Mejora. En primer lugar los Mapas de contenidos y Problemas (MCyP) (Figura 1). En el primer mapa se detalla la relación de contenidos: conceptos relativos a la necesidad de renovar el aire interior; conocimiento de los distintos sistemas de renovación del aire; conceptos relacionados con las normativas de aplicación y conceptos relacionados con los elementos del diseño de los sistemas de ventilación y conocimiento de las herramientas procedimentales relativas al cálculo y dimensionado de los elementos del sistema. En el segundo mapa se trata el tema de las estructuras horizontales centrado en la tipología de forjados unidireccionales de hormigón armado (HA), comprensión de elementos y trazado gráfico. A estos dos se añade un tercero en el que se estudiarán en clase los detalles constructivos más representativos que definen un forjado unidireccional de Hormigón Armado. 


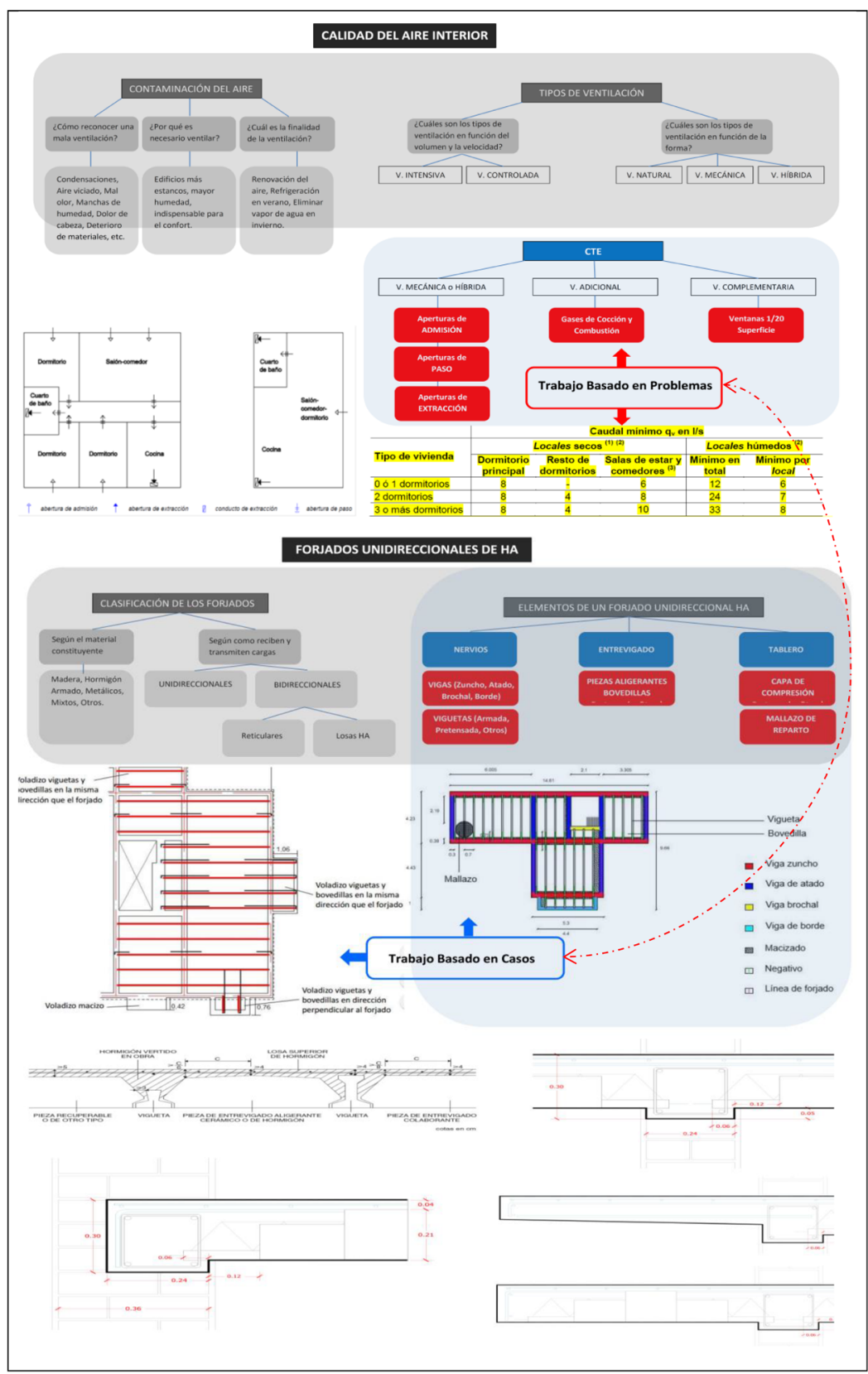

El Modelo Metodológico (figura 2) y la Secuencia de Actividades (tabla 1) y muestran en detalle la concatenación y organización de actividades en el CIMA.

Jornadas de Formación e Innovación Docente del Profesorado | № 2 (2019)

(C) Esta obra se distribuye con la licencia Creative Commons Reconocimiento-NoComercial-SinObraDerivada 


\section{Modificaciones introducidas en el CIMA del curso 2019-2020}

El Modelo metodológico usado en el CIMA sobre la misma asignatura en los pasados cursos 2017/18 y 2018/19 en cada uno de ellos presentó una serie de ventajas e inconvenientes. En el primero de ellos, 2017/18, es que se partía en cada sesión de un planteamiento inicial excesivamente reducido y se concedía mucho tiempo al trabajo en grupo. Esta dinámica no se mostró adecuada para todas las jornadas del CIMA, ya que el alumno no está acostumbrado a este tipo de actividades y posiblemente sea más práctica una introducción al trabajo autónomo más gradual. Por ello se propuso en el segundo CIMA, 2018/19, dividir las cuatro sesiones en dos bloques. Cada uno de ellos incluyendo una primera sesión de trabajo en la que el trabajo en grupo es más de tipo conceptual, relativo a los conceptos teóricos del ciclo, utilizando la herramienta de Aprendizaje Basado en Problemas (ABP) que requiere un menor nivel de información procedimental previo y que corresponde a las partes sombreadas en gris en el MCyP. Y una segunda fase en que se aplica ese contenido junto a una serie de herramientas gráficas para aplicarlos a procedimientos de cálculo y descripción de soluciones gráficas aplicadas a casos prácticos, utilizando para ello la herramienta de Aprendizaje Basado en Casos ( $A B C)$, y que corresponde a las partes sombreadas en azul en el MCyP (fig.1). Este esquema es el que se ha seguido en el CIMA del presente curso 2019/20.

Jornadas de Formación e Innovación Docente del Profesorado | № 2 (2019) Esta obra se distribuye con la licencia Creative Commons Reconocimiento-NoComercial-SinObraDerivada 


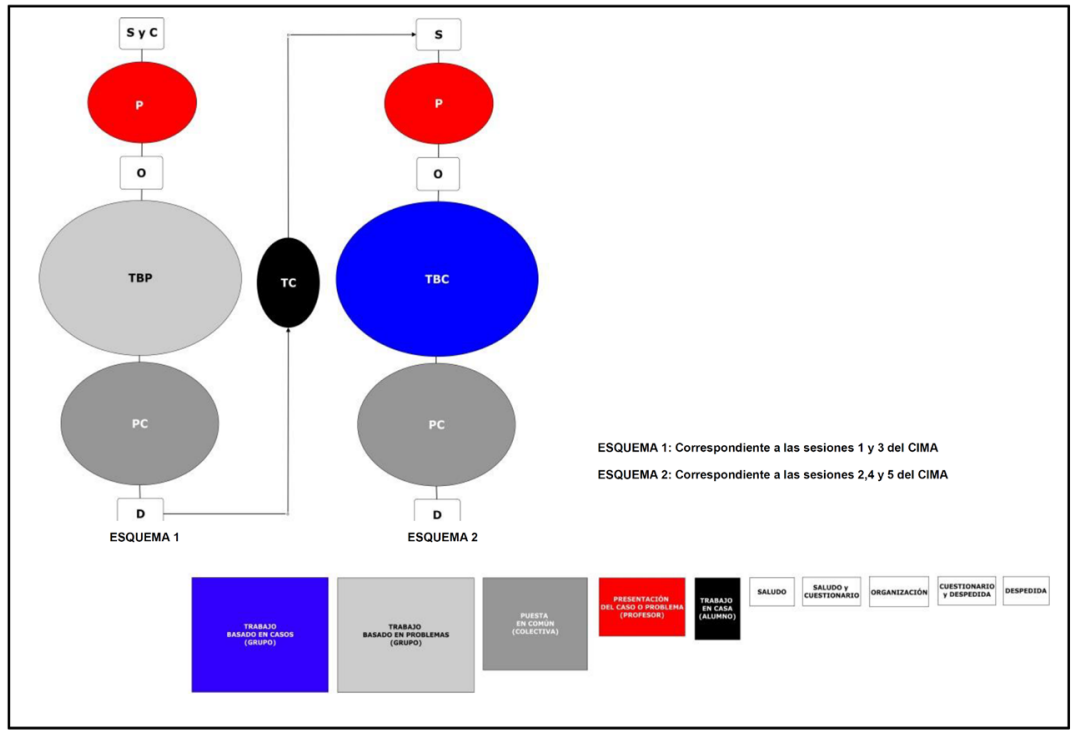

Figura 2. Modelo Metodológico y leyenda de actividades propuesta para el CIMA.

Como se puede observar en la tabla siguiente en la que se detalla la Secuencia de Actividades (tabla 1), el porcentaje de trabajo autónomo de los alumnos, siguiendo las pautas de Finkel [3], representa más del 70\% del total y las puestas en común un $15 \%$, por lo que las actividades en la que éstos no adoptan un rol protagonista se reduce a menos de un $15 \%$.

Tabla 1. Secuencia de Actividades propuesta para el CIMA.

\begin{tabular}{|c|c|c|c|}
\hline Actividad & Descripción & Tiempo & Sesión \\
\hline \multirow{2}{*}{ S y C } & $\begin{array}{l}\text { Saludo a los alumnos, repaso de los conceptos } \\
\text { fundamentales de la clase anterior, dudas sobre } \\
\text { ese contenido, descripción del contenido de la } \\
\text { clase presente, anuncios y comunicaciones sobre la } \\
\text { asignatura. Presentación del tema, contextualización } \\
\text { en la asignatura e índice. }\end{array}$ & $10^{-}$ & \multirow{3}{*}{$\begin{array}{l}\sigma \quad \\
m \\
> \\
\sigma \quad \\
r\end{array}$} \\
\hline & Cuestionario previo & $10^{-}$ & \\
\hline & $\begin{array}{l}\text { Información sobre el ciclo de mejora. Presentación } \\
\text { del Problema a desarrollar en clase. }\end{array}$ & 5 & \\
\hline
\end{tabular}




\begin{tabular}{|c|c|c|c|}
\hline 0 & $\begin{array}{l}\text { Conformación de grupos y Distribución del primer } \\
\text { Guion de Trabajo }\end{array}$ & 5 & \\
\hline TBP & Trabajo en grupos sobre el problema propuesto. & $55^{\prime}$ & \\
\hline $\mathbf{P C}$ & $\begin{array}{l}\text { Presentación de los grupos, comentarios y } \\
\text { correcciones. Recapitulación de contenidos } \\
\text { fundamentales y dudas finales. }\end{array}$ & 25 & \\
\hline D & $\begin{array}{l}\text { Descripción de la propuesta para el trabajo en casa } \\
\text { y despedida. }\end{array}$ & $10^{-}$ & \\
\hline $\mathbf{T C}$ & $\begin{array}{l}\text { Trabajo autónomo de los alumnos en casa sobre la } \\
\text { propuesta planteada. }\end{array}$ & $120^{-}$ & \\
\hline $\mathbf{S}$ & $\begin{array}{l}\text { Saludo a los alumnos, repaso de los conceptos } \\
\text { fundamentales de la clase anterior, dudas sobre } \\
\text { ese contenido, descripción del contenido de la } \\
\text { clase presente, anuncios y comunicaciones sobre la } \\
\text { asignatura. }\end{array}$ & 5 & I \\
\hline $\mathbf{P}$ & Presentación del Caso a desarrollar en clase. & 5 & \\
\hline 0 & $\begin{array}{l}\text { Conformación de grupos y Distribución del primer } \\
\text { Guion de Trabajo }\end{array}$ & 5 & $\frac{\sigma_{1}}{2}$ \\
\hline TBC & Trabajo en grupos sobre el caso propuesto. & $70^{-}$ & $\sigma i$ \\
\hline $\mathbf{P C}$ & $\begin{array}{l}\text { Presentación de los grupos, comentarios y } \\
\text { correcciones. Recapitulación de contenidos } \\
\text { fundamentales y dudas finales. }\end{array}$ & 25 & \\
\hline D & Comentarios finales y despedida. & $10^{-}$ & \\
\hline
\end{tabular}

\section{Material específico elaborado para el CIMA}

A partir del segundo CIMA, curso 2018/19, se comenzaron a elaborar apuntes específicos para el CIMA que sintetizan en bloques compactos de no más de cuatro páginas los temas, para ello se ha tenido en cuenta una jerarquización de contenidos sistemática. La idea es que los alumnos los tengan disponibles unos días antes de la clase y los utilizan para realizar los distintos ejercicios de las sesiones de trabajo siguiendo los respectivos Guiones de Trabajo en el aula. Se comprobó el curso pasado la efectividad de dicho recurso didáctico, por ello en el CIMA del presente curso se volverá a utilizar dicho material, 
ampliándose el mismo en relación al aumento en extensión del CIMA propuesto. Asimismo se mantienen los distintos modelos de edificios como base para la realización de ejercicios por grupos de alumnos en clase sobre distintos ejemplos de tal forma que se plantee un debate en la puesta en común de resultados a nivel colectivo (Figura 4).

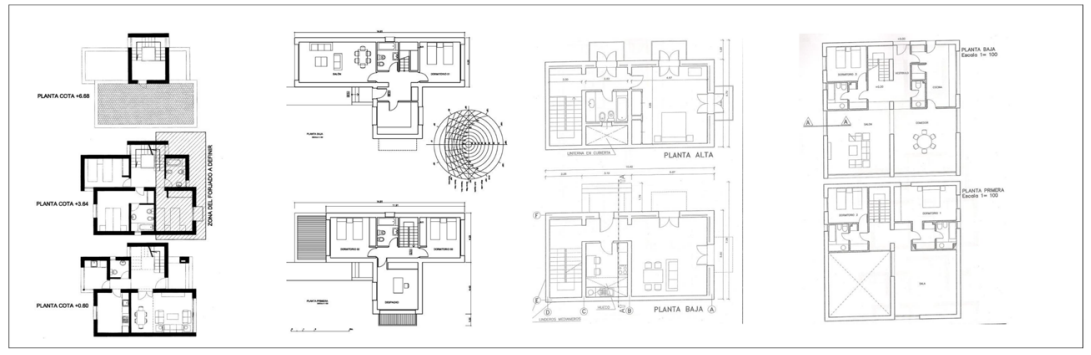

Figura 4. Ejemplos de algunos de los modelos de edificios utilizados para impartir el CIMA.

\section{Aplicación del CIMA}

\section{Relato de las Sesiones}

El CIMA se ha llevado a cabo en un grupo de unos treinta alumnos, en horario de tarde y con docencia los lunes y viernes en tres sesiones de dos horas. El siguiente Diario de Sesiones es un resumen de la experiencia que contiene referencias a lo vivido en el aula. Antes del comienzo del CIMA, los alumnos cumplimentaron cuestionarios específicos con preguntas teóricas y prácticas sobre los temas a tratar. Por primera vez se incluyeron preguntas gráficas, en concreto detalles constructivos que los alumnos tuvieron que identificar en cada uno de sus elementos.

Al ser similar el desarrollo de las cuatro sesiones del CIMA, con la salvedad de la inclusión del cuestionario en la primera y la última de ellas, es posible realizar una narración integrada del conjunto de las sesiones de trabajo 
del CIMA. En sintesis las sesiones han comenzado con una breve introducción al trabajo diario por parte del profesor y una explicación del Guion de trabajo en relación a la documentación remitida a los estudiantes unos días antes del comienzo de cada una de las sesiones. A continuación se ha procedido al trabajo autónomo en grupo, fase a la que ha sucedido una puesta en común en la que ha participado toda la clase, guiada por el profesor, en la que se han subrayado los contenidos más importantes abordados en cada una de las sesiones. La culminación de la clase ha sido una indicación a la siguiente sesión en cuanto a contenidos y trabajo a realizar, anunciando a los alumnos el día en que tendrán disponibles los apuntes de trabajo previos a la sesión. La fluidez del transcurso de las sesiones, la concentración de los estudiantes durante la sesión, la ausencia de tiempos muertos y la participación de todos los integrantes de cada grupo han generado un clima de trabajo mantenido durante toda la duración del CIMA.

\section{Evaluación del Aprendizaje de los Estudiantes}

El apartado de la Evaluación es uno de los principales recursos para validar de forma objetiva la efectividad del CIMA, de ahí que resulta clave el diseño de un sistema que permita la verificación precisa tanto a nivel individual como colectivo del grado de cumplimiento de los objetivos iniciales del aprendizaje. A pesar del incremento del éxito en cuanto a porcentaje de aprobados por curso respecto a años anteriores de los alumnos que realizaron el CIMA el curso pasado, este es uno de los aspectos que han presentado una mayor complejidad en ediciones previas de CIMA en la asignatura. Por ello, y debido a la naturaleza de los contenidos y procedimientos abordados en el CIMA, se propone un ejercicio teórico-práctico inicial, no solo teórico, para valorar el estado previo (fig. 5). Dicho ejercicio abordará una síntesis de los principales contenidos, tanto conceptuales como procedimentales, que se tratarán en el 
CIMA. Asimismo se propone una valoración específica no solo para cada tema, sino para valorar de forma aislada la permeabilidad relativa del estudiante hacia contenidos teóricos y prácticos de cada uno de ellos.

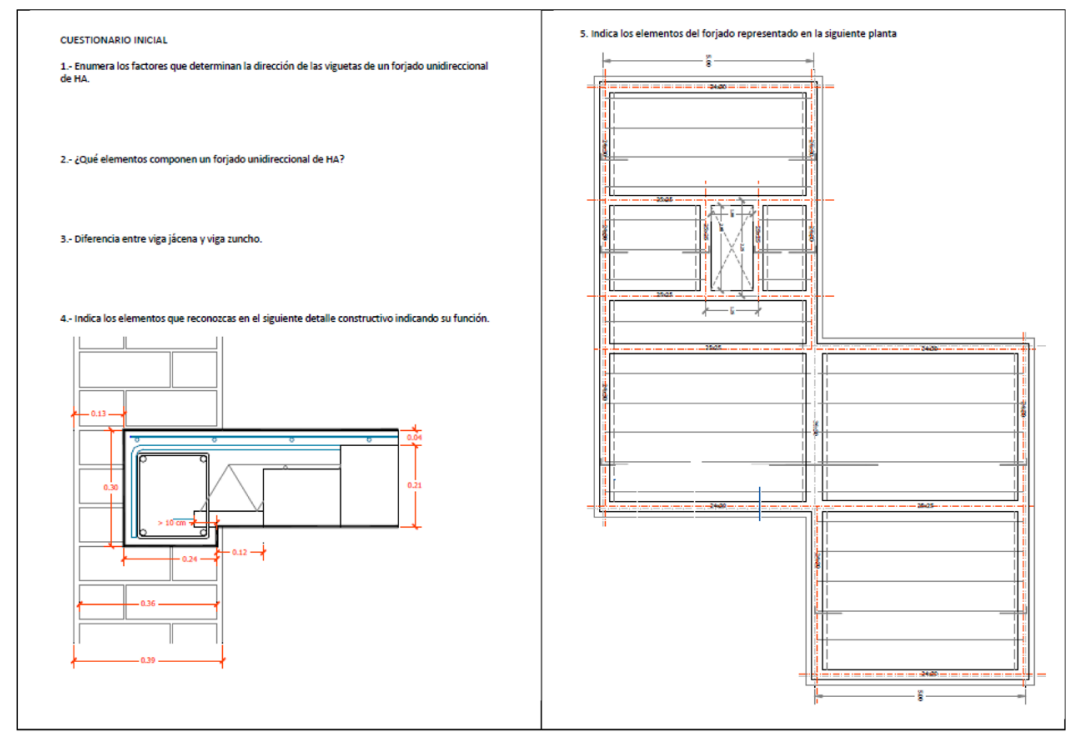

Figura 5. Ejemplo de uno de los cuestionarios inicial y final del CIMA.

La muestra considerada para el estudio fue un total de 24 encuestas individuales. Se ha unificado en una única Escalera el conjunto de las respuestas a las nueve preguntas incluidas en los Cuestionarios. En la siguiente gráfica (fig. 6) se muestran los porcentajes de los alumnos que se encuentran en cada peldaño, identificando a qué contenido se refiere cada nivel. Del análisis de estos resultados se desprende las dificultades relativas de aprendizaje para pasar de un nivel a otro superior y para obtener un grado entre adecuado y óptimo de conocimientos en relación a la materia abordada en el CIMA. En color azul la escalera inicial y en rojo la final. Trazándose la línea de nivel de conocimientos adecuados se observa que, antes del CIMA, este nivel estaba en un 29,5\%, debido fundamentalmente a la presencia de alumnos repetidores o procedentes de Ciclos Formativos en Edificación. Después del CIMA este 

al nivel de conocimientos satisfactorios. Lo cual implica una alta potencialidad para superar dicho nivel.

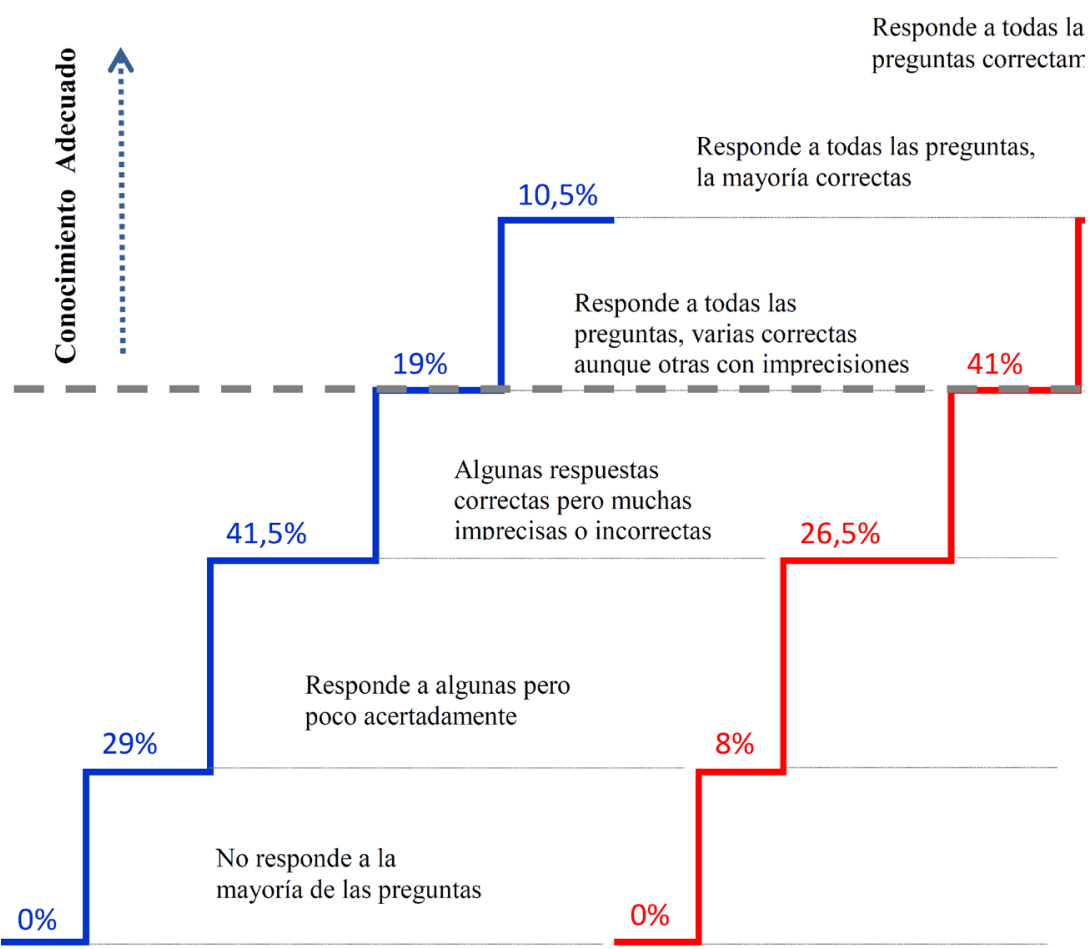

Figura 6. Escalera de Conocimiento y Aprendizaje elaborada en base a las respuestas a los Cuestionarios Inicial y Final.

Jornadas de Formación e Innovación Docente del Profesorado | № 2 (2019) Esta obra se distribuye con la licencia Creative Commons 


\section{Evaluación del Diseño puesto en práctica}

\section{Avances Conseguidos en el Ciclo de Mejora.}

El CIMA ha permitido romper la dinámica habitual de la clase magistral a la que están acostumbrados los alumnos, les ha obligado a realizar un trabajo intelectual poco frecuente en clase, les ha dado la oportunidad de participar y expresar sus propias ideas, trabajar cooperativamente en grupo y a actuar proactivamente con un alto aprovechamiento del tiempo. Las clases han resultado en general más amenas e interesantes y la secuencia de las distintas actividades ha propiciado un dinamismo mayor y una notablemente más alta conexión de los estudiantes con la materia tratada en las distintas sesiones. Las relaciones profesor-estudiante y estudiantes entre sí se han reforzado en confianza tras la experiencia del CIMA, especialmente en las actividades de trabajo en grupo y puesta en común que han ocupado la mayor parte del tiempo. Se ha conseguido, además, que los estudiantes lleguen al aula con una información previa y un conocimiento aproximado de los ejercicios a tratar durante esta fase. Lo cual ha eliminado la necesidad de largas introducciones para situarles en disposición de abordar los problemas y casos planteados. El nivel de concentración del grupo ha sido sorprendentemente alto durante todas las sesiones y ello se ha reflejado no sólo en los resultados el Cuestionario fina, sino en los distintos ejercicios realizados en clase. Desde la primera sesión se han mostrado proclives a probar una nueva metodología que les concediese más protagonismo dentro del aula y esta actitud se ha ido reforzando con el avance de las sesiones el CIMA. 


\section{Dificultades Encontradas}

La mayor dificultad ha consistido en concebir y elaborar un material específico para las distintas sesiones del CIMA. En este sentido, la experiencia en Ios CIMA realizados el curso 2018/19 ha posibilitado ajustar este nuevo material, tanto en los modelos, como en la información previa a cada clase, como en los guiones de trabajo de cada sesión. Para ello se han simplificado los Mapas de Contenidos y Problemas destacando aquellos contenidos fundamentales y adecuando el trabajo en las sesiones a las distintas zonas del mapa. También se han elaborado resúmenes específicos de los temas que incluyes aspectos esenciales de éstos y relacionados directamente con el trabajo a realizar en la sesión inmediata. Se han seleccionado diferentes modelos de edificios para cada uno de los grupos de trabajo, adecuados todos ellos a los criterios de la tipología constructiva tratada en la asignatura. A través de un mismo modelo para cada grupo a lo largo de todo el CIMA, el estudiante ha podido irse familiarizando con él y poner en relación aspectos relativos a los dos temas tratados en el CIMA. Por último, se han diseñado Guiones de Trabajo suficientemente compactos para no diversificar excesivamente las cuestiones a resolver englobando, al mismo tiempo, todas las cuestiones relevantes, tanto teóricas como procedimentales, de cada tema siguiendo las directrices recogidas en el texto Enseñanza universitaria. Como mejorarla [4]. En resumen, las dificultades encontradas han estado más fuera que dentro del aula, en la preparación del Ciclo, no en el transcurso del mismo.

\subsection{Aspectos a Mantener como Cambios Habituales}

El CIMA en su conjunto ha sido de gran utilidad, las nuevas técnicas didácticas implementadas han dinamizado la clase haciendo posible un mejor aprovechamiento de la 
misma. La aplicación del CIMA ha trascendido la función meramente transmisora de contenidos para favorecer las dinámicas internas del grupo en las relaciones entre estudiantes y de profesor y estudiantes. Mejorando el clima general en clase y estimulando la motivación de los estudiantes hacia la asignatura. Todas las herramientas didácticas empleadas en el desarrollo del CIMA merecen ser tenidas en consideración en el futuro, mejorándose sucesivamente según el aprendizaje derivado de la experiencia de su puesta en práctica. Es un enorme salto desde el planteamiento previo de la docencia de la asignatura, de hecho, con bastante probabilidad, tanto alumnos como profesor echaremos de menos esta dinámica en el resto de temas de la asignatura fuera del CIMA. Dada la efectividad demostrada en el desarrollo del CIMA del material específico elaborado, el propósito que surge a partir de aquí es seguir desarrollando dicho material para el resto de temas de la asignatura de tal forma que sea un proceso relativamente sencillo la extensión del Ciclo al conjunto de la misma.

\section{Conclusiones Finales}

El CIMA sirve para poner en crisis el sistema habitual basado esencialmente en el modelo de clases magistrales con alguna incursión en la solución de problemas. Las ventajas derivadas de transferir la responsabilidad de la clase a los estudiantes ha resultado sorprendentemente fácil y de incuestionable utilidad en términos de rendimiento del tiempo de trabajo efectivo. Esta dinámica, nueva para ellos, les sorprende y activa intelectualmente, lo cual supone un aprendizaje directo e intensivo. Por otro lado permite que el estudiante establezca una conexión personal con los contenidos. Respecto al profesor, la preparación de la clase es más trabajosa y compleja, pero su desarrollo más efectivo y gratificante. La definición del planteamiento de problemas y casos en los Guiones de Trabajo en 
base a los contenidos determinados como estructuradores en base a un análisis concienzudo del tema para conectarlo con la práctica profesional y establecer una estructura coherente de dichos contenidos en el Mapa general que los conecta es determinante para encajar la materia en una dinámica que dé el protagonismo al estudiante. En síntesis, una experiencia docente de enorme utilidad tanto en el plano puramente de transmisión de la información como en el de incentivar la iniciativa de los estudiantes, su compromiso con la materia y sus capacidades reflexivas, de trabajo individual y en equipo.

Jornadas de Formación e Innovación Docente del Profesorado | № 2 (2019) Esta obra se distribuye con la licencia Creative Commons 


\section{Bibliografía}

[1] Código Técnico de la Edificación. CTE. Disponible en la web: https: / www.codigotecnico.org/ (Última vez consultado Octubre 2018).

[2] Blain, K. (2007). Lo que hacen los mejores profesores de Universidad. Publicaciones de la Universitat de Valéncia. Valencia, España. ISBN: 9788437066677

[3] Finkel, D. (2008). Dar clase con la boca cerrada. Publicaciones de la Universitat de Valéncia. Valencia, España. ISBN: 978-84-370-7268-5

[4] Porlán, R. (Coord.). (2017). Enseñanza universitaria. Cómo mejorarla. Madrid: Ediciones Morata. 